\title{
Estudio sobre autoestima de estudiantes de nivel superior
}

\section{Study on self-esteem of upper level students}

\author{
SESENTO, Leticia $^{1} \&$ LUCIO, Rodolfo ${ }^{2}$ \\ ${ }^{1}$ Colegio Primitivo y Nacional de San Nicolás de Hidalgo \\ ${ }^{2}$ Facultad de Medicina Veterinaria y Zootecnia de la Universidad Michoacana de San Nicolás de Hidalgo
}

ID $1^{\text {er }}$ Autor: Leticia, Sesento / ORC ID: 0000-0002-6456058X, Researcher ID Thomson: S-6997-2018, CVU CONACYT ID: 449302

ID $1^{\mathrm{er}}$ Autor: Rodolfo, Lucio / ORC ID: 0000-0002-0535-3652, Researcher ID Thomson: X-2391-2018

DOI: $10.35429 / J U M .2019 .9 .3 .15 .20$

Recibido 12 de Junio, 2019; Aceptado 30 de Septiembre, 2019

\section{Resumen}

Este artículo trata sobre la autoestima como un aspecto de enorme relevancia en la vida de la persona, por cuanto contribuye a que su existencia sea más satisfactoria. La investigación es de corte cuantitativo descriptivo. El objetivo es conocer el nivel la autoestima en los estudiantes nivel superior. Para lo cual se aplicó el instrumento IAME a 148 estudiantes. En el análisis se observa que los alumnos manejan un alto nivel de autoestima debido a que los jóvenes de educación superior muestran un nivel (85\%), lo cual, puede ser producto de la edad, el entorno familiar y las características personales, sin embargo un $15 \%$ de la población encuestada muestra dificultades en el autoestima, esta población es el punto a trabajar como institución debido a que al tener baja autoestima no sólo repercute en la personalidad sino en el desarrollo de las diversas actividades académicas lo cual conlleva a un bajo rendimiento académico, es por ello que se propone implementar estrategias de orientación a los universitarios sobre el impacto en los diversos aspectos de la vida que tiene una baja autoestima y proponer una canalización de ser necesario a un especialista.

\section{Autoestima, Nivel Superior}

\begin{abstract}
This article deals with self-esteem as an aspect of enormous relevance in the life of the person, inasmuch as it contributes to their existence being more satisfactory. The investigation is of a descriptive quantitative cut. The objective is to know the level of self-esteem in the upper level students. For which the IAME instrument was applied to 148 students. In the analysis it is observed that the students manage a high level of self-esteem because the young people of higher education show a level $(85 \%)$, which can be a product of age, family environment and personal characteristics, however $15 \%$ of the surveyed population shows difficulties in self-esteem, this population is the point to work as an institution because having low self-esteem not only affects the personality but the development of various academic activities which leads to a low academic performance, that is why it is proposed to implement guidance strategies for university students on the impact on the various aspects of life that has low self-esteem and propose a channeling if necessary to a specialist.
\end{abstract}

\section{Self-esteem, Upper level}

Citación: SESENTO, Leticia \& LUCIO, Rodolfo. Estudio sobre autoestima de estudiantes de nivel superior. Revista de Gestión Universitaria. 2019. 3-9: 15-20

\footnotetext{
*Correspondencia del Autor (correo electrónico: leticiasesentogarcia@yahoo.com.mx)

$\dagger$ Investigador contribuyendo como primer autor.
} 


\section{Introducción}

La autoestima es una imagen personal que se va formando a lo largo de toda la vida, en la adolescencia la percepción y valoración que tiene el joven de sí mismo resulta un punto muy importante para lograr una de las tareas más esenciales que es la formación de una identidad, al igual que ésta identidad la autoestima consta de varios elementos, por lo que podemos decir que es multidimensional, ya que reúne aspectos de tipo social, moral, físico, cognitivo y sexual. Al final de la adolescencia aparece la capacidad para coordinar, resolver y normalizar los atributos contradictorios, y se reduce la experiencia de conflicto sobre el tipo de gente que la persona realmente quiere ser (Harter y Monsour, 1992, citado en Rice 1997).

Otros autores como Rodríguez y Caño (2012) consideran que los niveles de autoestima son estables durante la infancia, sin embargo, en la adolescencia las variaciones comienzan a emerger. Según estos autores, los hombres que consiguen logros personales presentan una autoestima alta, mientras que la autoestima de las mujeres depende de las percepciones de los demás.

Por otro lado, Silva-Escoria y MejíaPérez (2015) en un estudio en México, concluyen que los adolescentes con baja autoestima son propensos a un bajo rendimiento académico y comportamientos improductivos en algunas áreas de la esfera social. Es por esta repercusión en varios aspectos de la vida del joven que se debe poner atención en ésta temática en nuestros estudiantes.

\section{Marco Teórico}

La autoestima influye directamente en el comportamiento de los individuos e incide en la promoción del bienestar psicológico, pues la forma en que se evalúa a sí mismo el individuo tiene repercusión en todas las áreas del desarrollo social, emocional, intelectual, conductual y escolar (Herrero, 1994; González Arratia et al., 2000). Lo anterior cobra relevancia al considerar que le corresponde a la universidad actual preparar seres humanos integrales, mediante el desarrollo de aptitudes y la promoción de actitudes para que a nivel profesional sean capaces de resolver con éxito los problemas sociales.
La universidad tiene además el compromiso de identificar en sus aspirantes el nivel de autoconocimiento, autocontrol y autoestima que permita al alumno apreciarse y asumir la responsabilidad de dirigir su vida, debido a que ello constituye las herramientas que le facilitan la armonía y la convivencia con el ambiente, así como también las destrezas cognitivas que le permitirán progresar en su carrera universitaria (Valbuena, 2002).

Se debe considerar que la autoestima se va formando a lo largo de toda la vida, en la adolescencia la percepción y valoración que tiene el joven de sí mismo toma un auge importante para lograr una de las tareas más esenciales que es el logro de la identidad, al igual que ésta identidad la autoestima consta de varios elementos, por lo que podemos decir que es multidimensional, ya que reúne aspectos de tipo social, moral, físico, cognitivo y sexual. Al final de la adolescencia aparece la capacidad para coordinar, resolver y normalizar los atributos contradictorios, y se reduce la experiencia de conflicto sobre el tipo de gente que la persona realmente quiere ser (Harter y Monsour, 1992 citado en Rice 1997).

\section{Componentes de la autoestima.}

De acuerdo con Gastón de Mézerville (2004) existen una serie de aspectos interrelacionados que componen la autoestima. A partir de esta concepción, presenta un modelo propio al que ha denominado proceso de la autoestima. En este, se considera a la autoestima según dos dimensiones complementarias, una actitudinal inferida integrada por tres componentes: la autoimagen, la autovaloración y la autoconfianza. Los cuales se explican a continuación. La autoimagen se refiere a la capacidad de verse a sí mismo como la persona que realmente es, con sus virtudes y defectos. La autovaloración se refiere al respeto que se tiene cada persona al sentirse digna de los aspectos positivos que le pasan. Y por último, la autoconfianza se refiere a la capacidad de la persona de creer que puede realizar bien distintas cosas y sentirse segura al hacerlas.

En cuanto a la dimensión conductual observable, ésta también se encuentra conformada por otros tres componentes: el autocontrol, la autoafirmación y la autorrealización. 
El autocontrol, se refiere a la capacidad de sentirse capaz de controlar y dirigir su propia conducta. Por otro lado, la autoafirmación, se refiere a poder tomar decisiones con autonomía y madurez. Y por último la autorrealización, hace referencia a vivir una vida satisfactoria al cumplir los objetivos propuestos.

\section{Características de la alta y baja autoestima}

Molina, Baldares y Maya (1996, p. 30) describen a las personas con alta autoestima de la siguiente manera: Una persona con alta autoestima no se considera a sí misma como el centro del universo y mejor que los demás y las demás. Simplemente conoce muchos aspectos de sí misma, tiene autorespeto y tiene consciencia de su propio valor como persona única e irrepetible. Reconoce sus cualidades buenas, pero no se cree perfecta.

$\mathrm{Al}$ contrario, puede ser que tenga mucha consciencia sobre sus defectos y del hecho de que ella comete errores. Pero no ve sus defectos y errores como representativos de alguien sin valor, ella los entiendo como representativos del ser humano.

Por otra parte, según lo señalan Rosenthal y Simeonsson en Rice (2000), las personas con baja autoestima muestran una identidad cambiante, inestable y abiertamente vulnerable a la crítica o al rechazo, lo que verifica su inadecuación, incompetencia y falta de valía.

Puede que se perturben profundamente cuando piensan que se ríen de ellas, cuando sienten que las acusan o cuando perciben que otras personas tienen una opinión negativa de sí mismas. Cuanto más vulnerabilidad experimentan, mayores son sus niveles de ansiedad. Como resultado, se sienten torpes e intranquilas y evitan a toda costa exponerse al ridículo.

Santrock (2002) comenta que para muchos estudiantes y para muchas estudiantes, la autoestima baja puede ser una situación temporal. Sin embargo, para algunas y para algunos de ellos la autoestima se puede traducir en otros problemas más serios. Una pobre y persistente autoestima puede estar relacionada con el bajo rendimiento académico, la depresión y otras situaciones como la delincuencia.

\section{Metodología}

Se encuestó a 148 alumnos con un rango de edad de los 18 a los 46 años. Se utilizó el instrumento "Cuestionario de evaluación de autoestima", la cual, es el producto de la reducción de una prueba llamada IAME, que consta de 19 reactivos diseñados en las dimensiones físicas, general, competencia académica, emocional, relaciones con otros, 16 de ellos miden autoestima y los otros 3 miden autocrítica. Con opciones de respuesta de 1 muy de acuerdo, 2 algo de acuerdo, 3 algo en desacuerdo y 4 muy en desacuerdo.

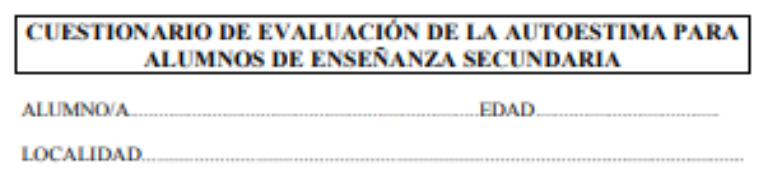

Seguidansente encontraris una serie de frases en las cuales se hacen afirmaciones relacionadas con tu forma de ser y de sentinte. Después de leer cada frase, rodea con un circulo hapción de respuesta (A, B, C, o D) que exprese mejor ta grado de acuerdo con lo que, en cada frase se dice.

A- Mury de acuendo.

C-Algo de acuendo.

D-Muy en descuererdo.

\begin{tabular}{|c|c|c|c|c|}
\hline 1.- Hago muchas cosas mal. & A & $\mathrm{B}$ & $\mathrm{C}$ & D \\
\hline 2.- A menudo el profesor me llama la alención sin rabón. & A & $\mathrm{B}$ & c & D \\
\hline 3.- Me enfado algunas veces. & A & $\mathrm{B}$ & c & $\mathrm{D}$ \\
\hline 4.- En conjunto, me siento satisfecho conmigo mismo. & A & $\mathrm{B}$ & C & D \\
\hline 5.- Soy un chicola guapo/a. & A & $\mathrm{B}$ & c & D \\
\hline 6.- Mis podres estin contemlos con mis notas. & A & $\mathrm{B}$ & c & D \\
\hline 7.- Me gusta toda la gente que conozco. & A & B & $\mathrm{c}$ & D \\
\hline 8.- Mis podres me exigen demasisdo en los estudios. & A & $\mathrm{B}$ & $\mathrm{c}$ & $\mathrm{D}$ \\
\hline 9.- Me pongo nervioso cuando tenemos examen. & A & $\mathrm{B}$ & $\mathrm{c}$ & $\mathrm{D}$ \\
\hline \multicolumn{5}{|l|}{ 10.- Pienso que soy un chico'a listo/a. } \\
\hline 11. A veces tengo ganas de decir tacos y palabrotas. & $\mathrm{A}$ & $\mathrm{B}$ & $\mathrm{c}$ & $\mathrm{D}$ \\
\hline 12. Creo que tengo un buen número de buenas cualidades. & A & $\mathrm{B}$ & C & D \\
\hline 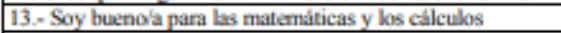 & A & $\mathrm{B}$ & C & $\mathrm{D}$ \\
\hline 14.- Me gustaria cambiar algunas partes de mi cuerpo. & A & $\mathrm{B}$ & c & $\mathrm{D}$ \\
\hline 15.- Creo que tengo un buen tipo. & $\mathrm{A}$ & $\mathrm{B}$ & C & $\mathrm{D}$ \\
\hline $\begin{array}{l}\text { 16.- Muchos de mis compañeros dicen que soy toope para } \\
\text { los estudios. }\end{array}$ & A & $\mathrm{B}$ & c & D \\
\hline \begin{tabular}{|l}
17 - Me pongo nervioso/a cuando me pregunta el profesor \\
\end{tabular} & A & $\mathrm{B}$ & $\mathrm{c}$ & $\mathrm{D}$ \\
\hline $\begin{array}{l}\text { 18.- Me siento inclinsdo a pensar que soy un'a fracasado'a } \\
\text { en todo. }\end{array}$ & A & $\mathrm{B}$ & c & $\mathrm{D}$ \\
\hline 19. Normalmente olvido lo que aprendo. & A & $\mathrm{B}$ & C & D \\
\hline
\end{tabular}

\section{Resultados}

Sobre la pregunta "Creo que hago muchas cosas mal", el $28 \%$ está de acuerdo y un $72 \%$ no, lo cual, nos permite observar que sienten que no cometen bastantes errores, situación de seguridad característica de alta autoestima. 


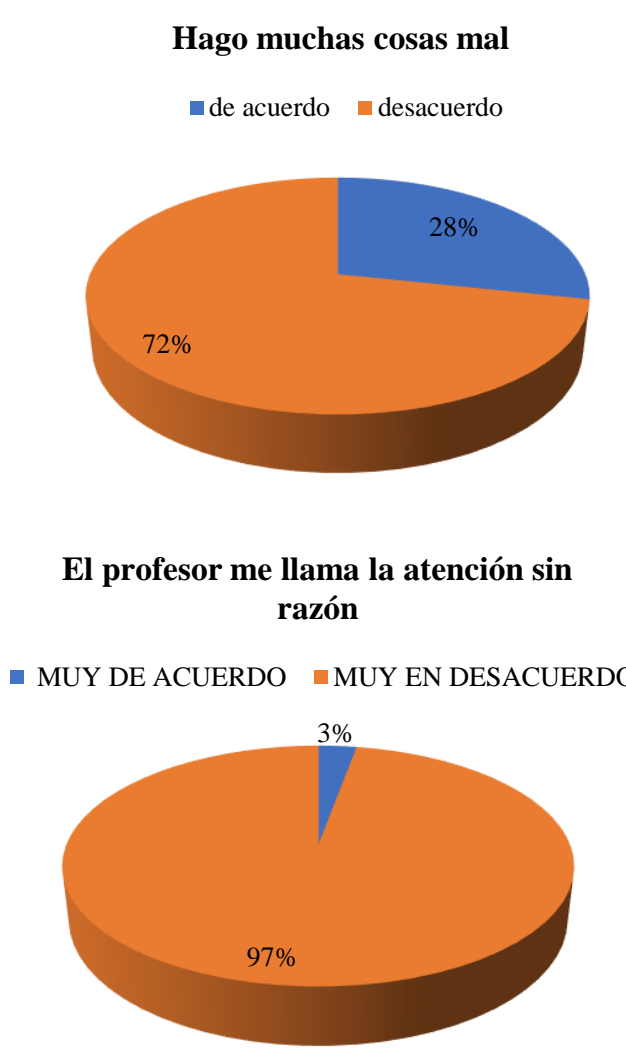

Esta segunda pregunta permite corroborar el nivel de autoconfianza y autovaloración, por lo que, se observa que no culpan a nadie de sus acciones ni se sienten acosados por profesores.

\section{Me enfado alguna veces}

- MUY DE ACUERDO MUY EN DESACUERDO

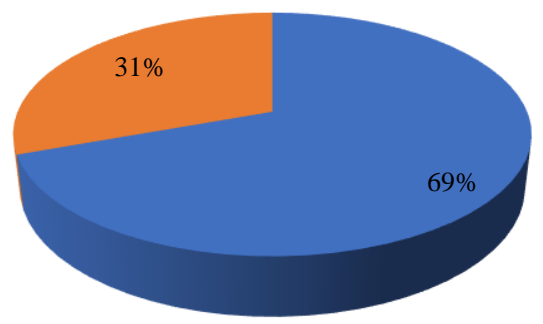

Esta pregunta permite conocer los niveles de autocontrol, y se observa que un gran porcentaje si se enfada pero se controla

me siento satifecho conmigo mismo

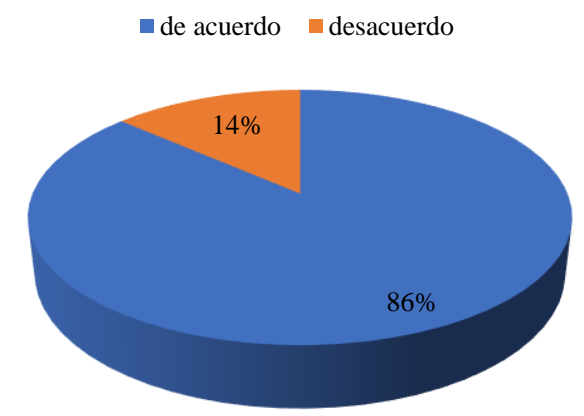

Sobre la cuestión "Me siento satisfecho conmigo mismo" una gran mayoría $86 \%$ se muestra contento consigo mismo, sin embargo, es relevante considerar el $14 \%$ que no lo hace.

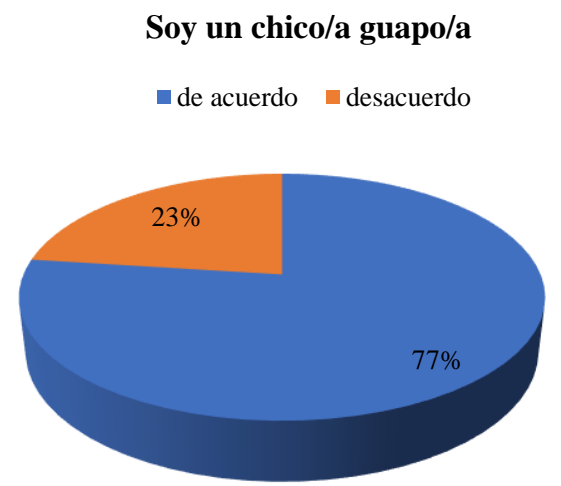

Analizando la tabla anterior se observa que un satisfactorio $77 \%$ se percibe atractivo físicamente y un $23 \%$ no se siente atractivo con su apariencia actual.

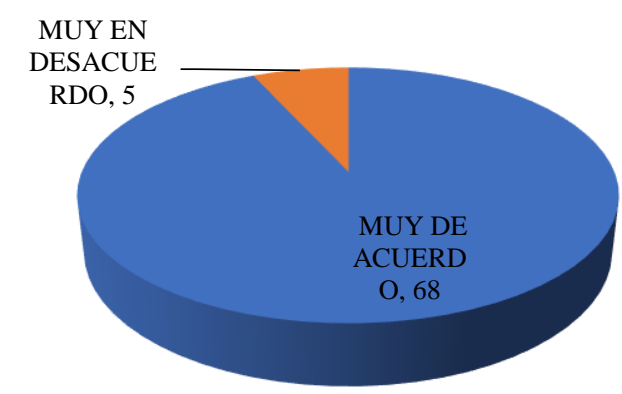

Esta grafica representa la pregunta "Mis padres están contentos con mis notas" y se observa que una parte importante se siente satisfecho y 5 de ellos no lo están

Mis padres me exigen demasiado

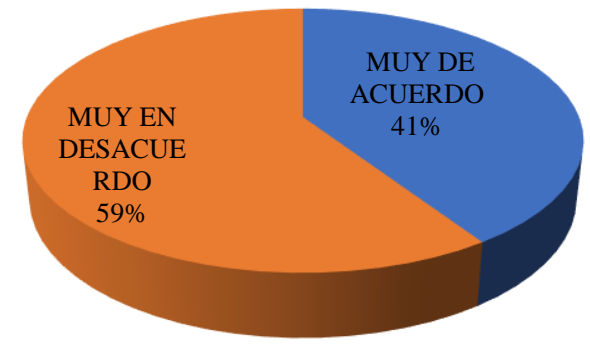

Ésta pregunta permite observar la autoimagen y autocontrol, un porcentaje considerable se siente presionado por sus padres, lo cual, puede repercutir en su autoconfianza. 


\section{Me pongo nervioso durante un exámen}

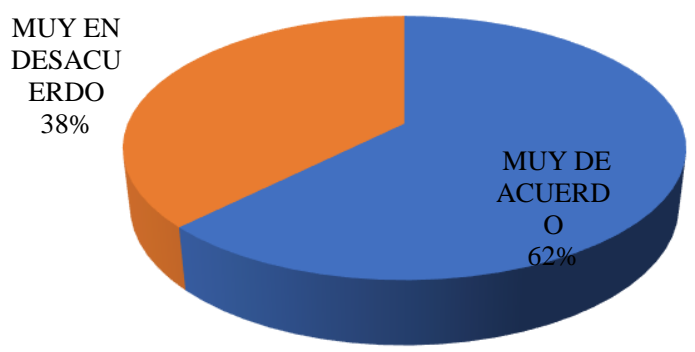

Esto es muy común en los estudiantes, pero puede ser indicador de autoconfianza y seguridad, podemos observar que una mayoría $62 \%$ si se presiona durante un examen.

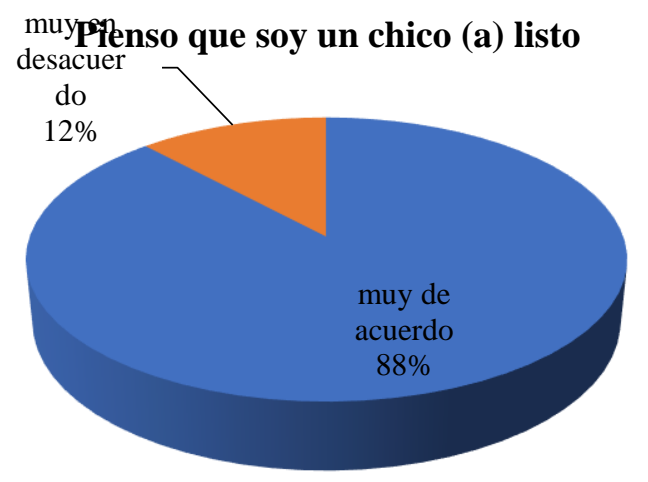

Esto permite observar la autoconfianza y la autoimagen de los estudiantes, y se observa que un importante $88 \%$ si se siente seguro de su capacidad para estudiar y lograr sus propósitos.

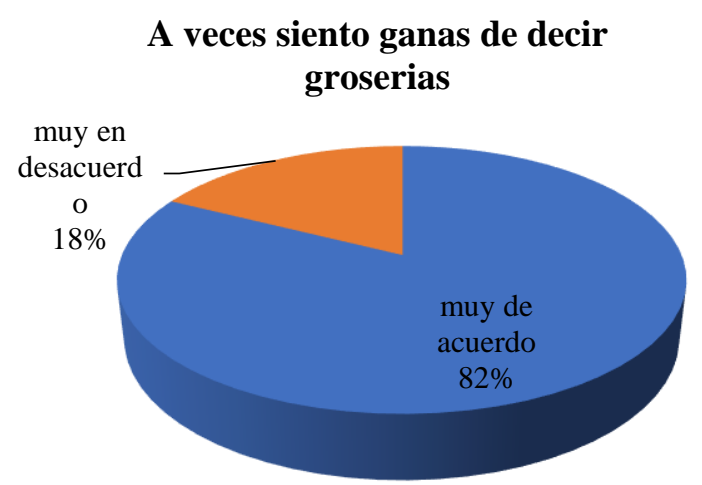

Esta pregunta nos ayuda a medir el autocontrol, pues si se sienten con la necesidad de maldecir, sin embrago, en pocas ocasiones llegan a hacerlo, se observa que un $82 \%$ si llega a sentirse muy molesto o frustrado.
Tengo un buen número de cualidades

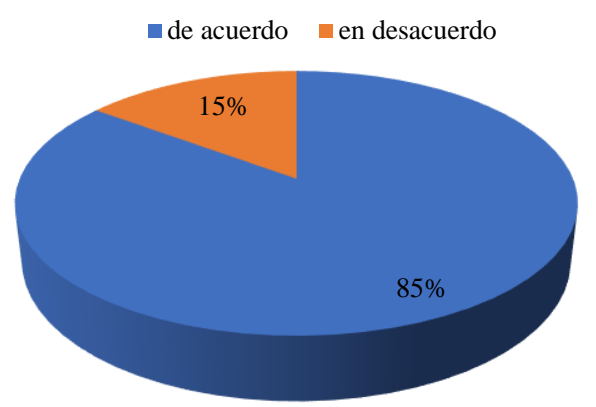

Sobre cómo perciben sus cualidades un $85 \%$ de los estudiantes encuestados se percibe con bastantes cualidades y un $15 \%$ considera no tener bastantes cualidades.

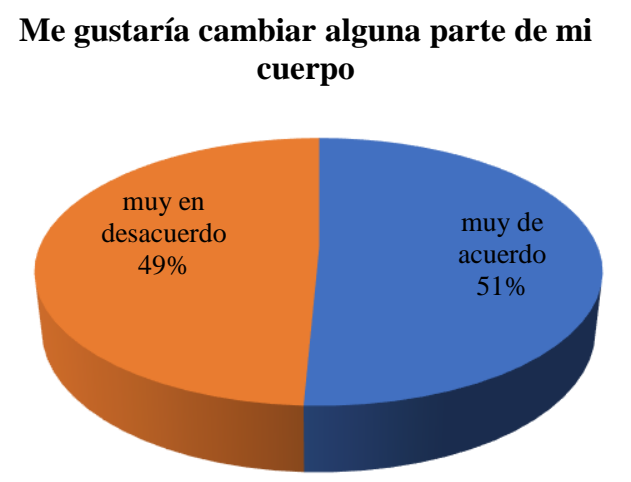

Esta es la pregunta más reñida, se refiere a la autoimagen y se observa que el $49 \%$ se encuentra satisfecho con su cuerpo y apariencia.

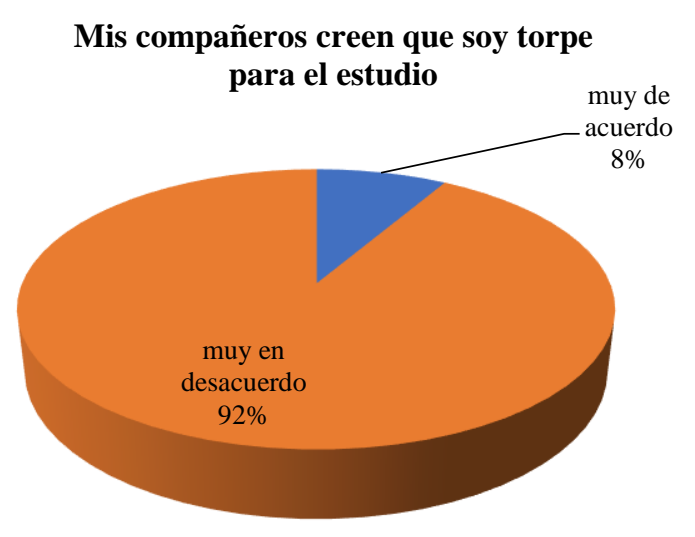

Un favorecedor $92 \%$ no se siente discriminado o juzgado negativamente por sus compañeros de clase. 


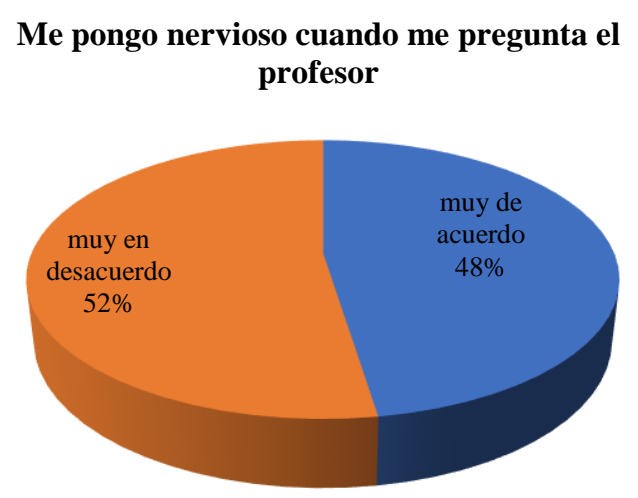

Al igual que en la pregunta del examen, esto puede ser "normal" sin embargo, se observa que son pocos más los que no se sienten intimidados que los que sí lo hacen.

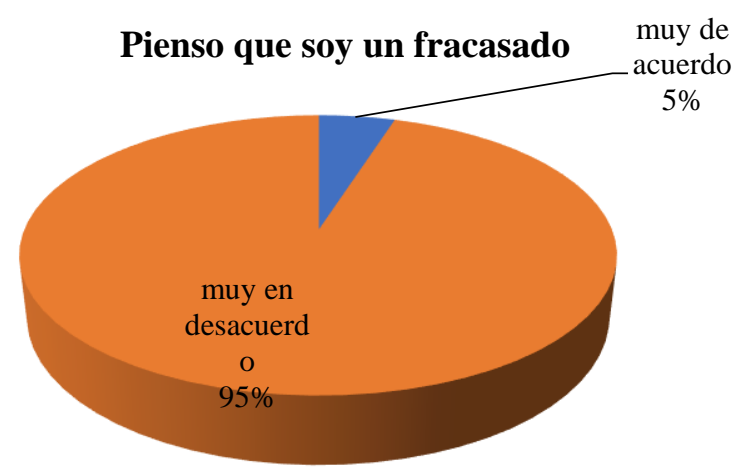

Resulta gratificante el porcentaje que se siente bien consigo mismo, sin embargo, se debe tomar en cuenta ese $5 \%$ que se siente fracasado para apoyar en medida de lo posible.

\section{Generalmente olvido lo que aprendo}

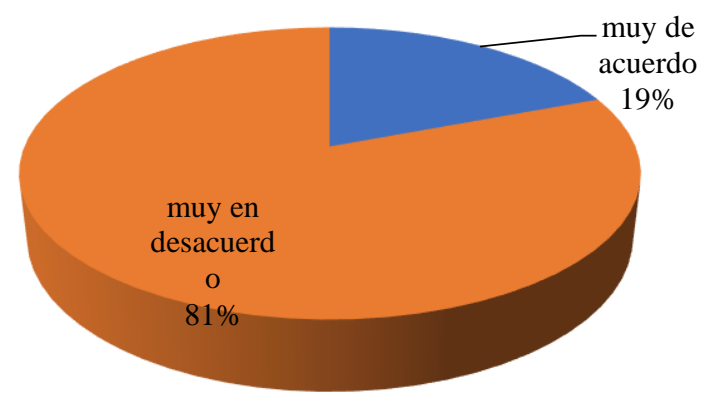

Se refiere a la seguridad en sus habilidades y capacidades lo cual, muestra que sólo el $19 \%$ se siente incapaz de retener la información aprendida.

\section{Conclusiones}

Realizando un análisis general de las gráficas se muestra que los jóvenes de educación superior muestran un nivel elevado de autoestima (85\%), lo cual, puede ser producto de la edad, el entorno familiar y las características personales, sin embargo un $15 \%$ de la población encuestada muestra dificultades en el autoestima, esta población es el punto a trabajar como institución debido a que al tener baja autoestima no sólo repercute en la personalidad sino en el desarrollo de las diversas actividades académicas lo cual conlleva a un bajo rendimiento académico, se necesita prestar atención en aquellos jóvenes que muestran índices bajos de seguridad, es por ello que se propone implementar estrategias de orientación a los universitarios sobre el impacto en los diversos aspectos de la vida que tiene una baja autoestima y proponer una canalización de ser necesario a un especialista. Con estas medidas se fortalece al joven y se puede reflejar en su desempeño y en los índices de deserción y reprobación.

\section{Referencias}

González-Arratia, N. I. (1996). El autoconcepto y la autoestima en madres e hijos, sus repercusiones en el rendimiento escolar, en niños de $6^{\circ}$ año de primaria, de tres diferentes tipos de escuela. Tesis de maestría, Facultad de Ciencias de la Conducta, Universidad Autónoma del Estado de México.

Herrero, J. (1994). Estresores sociales y recursos sociales: el papel de apoyo social en el ajuste bio-psico-social. Tesis doctoral. Universidad de Valencia, Valencia.

Molina, Margarita; Baldares; Thelma y Maya, Arnobio. (1996). Fortalezcamos la autoestima y las relaciones interpersonales. San José: Impresos Sibaja.

Santrock, John. (2002). Psicología de la educación. México: Mc Graw Hill.

Rice, F.P(1997). Desarrollo Humano. México: Prentice Hall Hispano americana.

Valbuena, B. A. (2002). http://www.revista candidus.com/secsi2/nuestras_htm.

(septiembre) 